\title{
Hunger is the best spice: an fMRI study of the effects of attention, hunger and calorie content on food reward processing in the amygdala and orbitofrontal cortex
}

Citation for published version (APA):

Siep, N., Roefs, A. J., Roebroeck, A. F., Havermans, R. C., Bonte, M. L., \& Jansen, A. T. M. (2009). Hunger is the best spice: an fMRI study of the effects of attention, hunger and calorie content on food reward processing in the amygdala and orbitofrontal cortex. Behavioural Brain Research, 198(1), 149-158. https://doi.org/10.1016/j.bbr.2008.10.035

Document status and date:

Published: 01/01/2009

DOI:

10.1016/j.bbr.2008.10.035

Document Version:

Publisher's PDF, also known as Version of record

Document license:

Taverne

Please check the document version of this publication:

- A submitted manuscript is the version of the article upon submission and before peer-review. There can be important differences between the submitted version and the official published version of record. People interested in the research are advised to contact the author for the final version of the publication, or visit the DOI to the publisher's website.

- The final author version and the galley proof are versions of the publication after peer review.

- The final published version features the final layout of the paper including the volume, issue and page numbers.

Link to publication

\footnotetext{
General rights rights.

- You may freely distribute the URL identifying the publication in the public portal. please follow below link for the End User Agreement:

www.umlib.nl/taverne-license

Take down policy

If you believe that this document breaches copyright please contact us at:

repository@maastrichtuniversity.nl

providing details and we will investigate your claim.
}

Copyright and moral rights for the publications made accessible in the public portal are retained by the authors and/or other copyright owners and it is a condition of accessing publications that users recognise and abide by the legal requirements associated with these

- Users may download and print one copy of any publication from the public portal for the purpose of private study or research.

- You may not further distribute the material or use it for any profit-making activity or commercial gain

If the publication is distributed under the terms of Article 25fa of the Dutch Copyright Act, indicated by the "Taverne" license above, 
Research report

\title{
Hunger is the best spice: An fMRI study of the effects of attention, hunger and calorie content on food reward processing in the amygdala and orbitofrontal cortex
}

\author{
Nicolette Siep ${ }^{\mathrm{a}, *}$, Anne Roefs ${ }^{\mathrm{a}}$, Alard Roebroeck ${ }^{\mathrm{b}}$, Remco Havermans ${ }^{\mathrm{a}}$, Milene L. Bonte ${ }^{\mathrm{b}}$, Anita Jansen ${ }^{\mathrm{a}}$ \\ a Department of Clinical Psychological Science, Faculty of Psychology and Neuroscience, Maastricht University, P.0. Box 616, 6200 MD Maastricht, The Netherlands \\ ${ }^{\mathrm{b}}$ Department of Cognitive Neuroscience, Faculty of Psychology and Neuroscience, Maastricht University, P.0. Box 616, 6200 MD Maastricht, The Netherlands
}

\section{A R T I C L E I N F O}

\section{Article history:}

Received 11 July 2008

Received in revised form 14 October 2008

Accepted 24 October 2008

Available online 6 November 2008

\section{Keywords:}

Attention

Calorie

Eating behaviour

fMRI

Satiety

Obesity

Incentive salience

Explicit processing

\begin{abstract}
A B S T R A C T
Research indicates that dysfunctional food reward processing may contribute to pathological eating behaviour. It is widely recognized that both the amygdala and the orbitofrontal cortex (OFC) are essential parts of the brain's reward circuitry. The aims of this fMRI study were (1) to examine the effects of food deprivation and calorie content on reward processing in the amygdala and the OFC, and (2) to examine whether an explicit evaluation of foods is necessary for OFC, but not amygdalar activity. Addressing the first aim, healthy females were presented with high and low calorie food pictures while being either hungry or satiated. For the second aim, attention focus was manipulated by directing participants' attention either to the food or to a neutral aspect. This study shows that hunger interacts with the energy content of foods, modulating activity in the posterior cingulate cortex, medial OFC, insula, caudate putamen and fusiform gyrus. Results show that satiated healthy females show an increased reward processing in response to low calorie foods. Confirming our hypothesis, food deprivation increased activity following the presentation of high calorie foods, which may explain why treatments of obesity energy restricting diets often are unsuccessful. Interestingly, activity in both the amygdala and mOFC was only evident when participants explicitly evaluated foods. However, attention independent activity was found in the MPFC following the high calorie foods cues when participants where hungry. Current findings indicate that research on how attention modulates food reward processing might prove especially insightful in the study of the neural substrates of healthy and pathological eating behaviour.
\end{abstract}

(c) 2008 Elsevier B.V. All rights reserved.
Research indicates that dysfunctional reward processing may be related to pathological eating behaviour [1-3]. In an environment where food is scarce, the reward system provides evolutionary benefits, because this system allows for consumption and storage of energy in the absence of homeostatic needs [4]. However, in western societies, food is highly accessible, energy-dense, heavily advertised and inexpensive. In this environment, the reward system poses a major health risk, predisposing many people to develop obesity $[5,6]$. Therefore, a strategy for understanding inappropriate or pathological eating behaviour is to identify factors that modulate food reward processes in the human brain.

It has been widely recognized that both the amygdala and the orbitofrontal cortex (OFC) play an important role in forming representations of environmental cues and their reward value [7-13]. The amygdala and the OFC are densely interconnected [14], and to

\footnotetext{
* Corresponding author. Tel.: +31 43 3881512; fax: +31 433884196

E-mail address: Nicolette.Siep@psychology.unimaas.nl (N. Siep).
}

carry out their reward functions they interact with other dopaminergic mesocorticolimbic structures, including the striatum, the dopaminergic midbrain and the anterior cingulate cortex [15]. It is hypothesized that the amygdala influences behaviour by providing a direct memory link between a stimulus and its incentive value. The amygdala then projects this information to the OFC, where this information is used to predict reward outcomes [16]. However the exact contributions of the amygdala and OFC to reward processing in general are still unknown.

Recent neuroimaging studies show a dysfunction of the mesocorticolimbic structures of obese people, which are similar to those observed in patients suffering from drug and alcohol addiction [17]. For example, it has been shown that when obese participants are exposed to food, this causes large and fast increases in mesolimbic dopamine release, similar to drug-cue elicited dopaminergic responses in drug addicts $[18,19]$. Furthermore, it has been shown that both patients suffering from cocaine addiction and obese people have a reduction in mesolimbic dopamine D2 receptors [20-22]. In turn this reduction in D2 receptors in obese people has been cor- 
related with a decreased metabolism in the orbitofrontal cortex [21]. The similar dysfunction of the mesocorticolimbic pathways in both patients suffering from addiction and obese people indicates similarities in their underlying pathology. Therefore knowledge about factors that can modulate mesocorticolimbic functioning might be especially insightful and aid in the development of effective treatments. However, little is known about factors that modulate reward processes in the brain, especially the OFC. Due to the vicinity to air-filled frontal and sphenoidal sinuses, OFC imaging is prone to artifacts, which complicates this kind of research [23]. Recently, investigators have developed specific methods to optimize imaging of the OFC [24]. Using these optimized methods, the current study investigates the influence of three modulating factors of reward processing in the OFC and the amygdala: calorie content, hunger, and attention.

A first hypothesized determinant of the regulation of reward processing is the calorie content of food [25]. According to the optimal foraging theory, organisms forage in such a way as to maximize their energy intake, which would provide evolutionary benefits [26]. Indeed, several studies indicate that people easily overeat of high calorie foods [27-29]. However, it is difficult to separate effects of calorie content and palatability as the two go often hand in hand. This notion is supported by studies finding little effect of calorie content on food intake, when controlling for palatability [30-32]. So far there has been only one neuroimaging study on the effect of calorie content on food reward processing [33]. In that fMRI study, participants had to memorize pictures of high calorie foods and low calorie foods inside the scanner for a later recollection task outside the scanner. The investigators found amygdalar activity following the presentation of the food pictures in contrast to non-food objects. However, this activity did not differ between high and low calorie foods. Furthermore, no activity in the OFC was found (but no optimizing techniques for OFC imaging were used), and differences in palatability ratings between the high and low calorie foods were not controlled for, which makes it hard to draw definite conclusions about the effect of caloric content per se on food reward processing.

A second factor that is hypothesized to influence reward processing is hunger. It is argued that people are motivated to eat due to the incentive salience of food, which elicits craving or 'wanting' [3,34-36]. However, these food reward processes are often complicated by the fact that the incentive salience of food depends, to some extent, on a co-occurring homeostatic state [5]. It is an old saying that hunger is the best spice, referring to the allesthesia phenomenon; that foods seem more attractive and palatable when hungry [37]. Yet it is still far from clear whether, how, and where in the human brain internal homeostatic processes and reward processes interact. Neuroimaging studies have indicated that the subjective evaluation of reward is highly correlated with activity in the OFC in monkeys [38] and humans [10]. Because hunger changes the subjective evaluation of foods, it has been hypothesized that the OFC plays an important role in the integration of hunger and incentive salience. In particular, it has been shown that OFC neurons in a monkey stop responding when fed to satiety [39]. Recent neuroimaging research $[9,10,40]$ has shown that feeding participants to satiety has a negative effect on the reported subjective pleasantness of the specific taste, a phenomenon referred to as sensory specific satiety (SSS). This decrease in pleasantness of food correlated highly with a reduction in activity of the OFC. However, it is currently unknown whether hunger, being the opposite of satiety, increases food reward activity in the OFC.

Furthermore, it can be hypothesized that hunger in interaction with calorie content affect food reward processes. For example, hunger might actually increase the rewarding value of high calorie foods more than that of low calorie foods. This idea is supported by a study of Gilhooly et al. [41], who investigated the relationship between craved foods and a 6-month dietary energy restriction. It was found that during the energy restriction participants craved energy-dense high-fat foods more than twice as much as before. It is important to further explore the relationship because energy restriction is one of the most applied strategies in the treatment of obesity [42]. The increase in reward value of energy-dense food might explain why obese people often ultimately fail in their attempts to lose weight after some initial success [43].

The third factor that is hypothesized to influence reward processing is attention. Although it has been proposed that both the amygdala and the OFC play an important role in reward evaluation, their exact contributions to reward processing in general remain unclear. Imaging studies of the amygdala commonly associate activation of this area with the perception of visual emotional stimuli, such as facial expressions [44,45] or unpleasant pictures [46]. A topic of current debate is the extent to which amygdalar responses are automatic [47]. Several studies report a stronger response of the amygdala during automatic emotional processing compared to explicit emotional evaluation [48]. It would be of interest to test whether food reward processing in the amygdala is also automatic and independent of attention focus. In contrast, OFC responses are believed to represent the explicit evaluation of food reward [49], highly dependent on attention focus. This might explain why previous neuroimaging studies of food reward using a passive task (i.e. a task that does not require explicit evaluation) failed to show OFC activity $[33,50,51]$. Therefore, the third objective of this study was to test whether amygdalar activity is related to the automatic processing of food cues, and whether OFC activity is related to the explicit evaluation of food.

In sum, the aims of this fMRI study were to examine the modulating effects of calorie content, hunger, and attention focus on reward processing in the amygdala and OFC of non-dieting female participants. Participants were presented with pictures of high and low calorie foods that were matched for palatability, during two fMRI sessions. Before one of the sessions, participants were offered a highly satiating lunch (containing $500 \mathrm{kcal}$ ), whereas in the other session participants were food deprived for $18 \mathrm{~h}$. It was hypothesized that high calorie foods would be more rewarding than low calorie foods, and that food deprivation would increase the reward value of food cues, especially the high calorie foods. Both effects were hypothesized to be reflected in an increase in the bloodoxygen-level dependent (BOLD) response in the amygdala and OFC. The question whether explicit evaluation of food palatability is necessary for OFC activity but not for amygdalar activity was addressed by manipulating the attention focus of the participants. It was hypothesized that directing the participant's attention to either the food or a neutral stimulus aspect, would result in a modulation of reward processing in the OFC but not in the amygdala. That is, food reward processing in the amygdala was expected to take place independent of attention focus.

\section{Method}

\subsection{Participants}

Female undergraduate students were recruited by flyers posted at Maastricht University. The students who applied for participation were invited for an interview in which height, weight, age, handedness, medication use, dietary restrictions, impulsivity, and reward responsiveness traits were assessed.

Selected participants were 12 right-handed, healthy students with a normal body weight (body mass index (BMI) between 18.5 and $25, M=21.5, S D=1.9$ ) and not currently dieting as assessed by self-report. Participants were unrestrained eaters, scoring $<15$ on the Restraint Scale $(M=8.9, S D=2.9)$ [52]. On average, participants were $19.3 \pm 0.9(M \pm S D)$ years old. Because food intake varies across the menstrual cycle in females [53], participants were selected based on the use of Combined Oral Contraceptives (COCs). COCs inhibit the production of fertility hormones and consequently prevent increases in food intake in the premenstrual phase [54]. Exclusion criteria were screened using a questionnaire and included items about personal or 
first-degree family history of eating disorders, depression or other psychiatric conditions and other illnesses that required a strict eating pattern (e.g., diabetes). Because certain personality traits are thought to reflect the sensitivity of the reward system [1], participants were screened on reward responsiveness and impulsivity. All participants scored within the normative ranges of impulsivity (participants' score: $M=63.3, S D=1.4$; normative score: $M=64.2, S D=10.7$ ) as measured with the Barratt Impulsiveness Scale [55]. In addition participants scored within the normative range of reward responsiveness (participants' score: $M=17.6, S D=2.1$; normative score: $M=17.5, S D=1.4$ ) as measured with a subscale from the BIS/BAS scale [56]. Written informed consent and ethical approval were obtained of each participant before the experiment. Participants completing the study received $€ 40$ compensation.

\subsection{Stimuli}

In a pilot study, 90 food and 40 non-food pictures were selected as candidate stimuli from an Internet database (www.istockphoto.com). Subsequently, these food pictures were rated by 15 healthy, non-dieting, female volunteers on 7-point Likert scales. The food pictures were rated on caloric content (1: very low-caloric - 7: very high-caloric) and palatability (1: very bad tasting -7 : very good tasting). The object pictures were evaluated on their emotional valence ( 1 : very negative associations 7: very positive associations). Based on these ratings, 15 food pictures rated as high in calorie content $(M=6.6, S D=0.3$; e.g., chocolate, pizza, hamburger $)$ and 15 food pictures rated low in caloric content $[M=1.5, S D=0.4$; e.g., cracker, melon, carrot paired $t$-test calorie ratings: $t(14)=56.60, P<0.001$ ], but judged as equally palatable $[t(14)=0.58, P=0.58$; high calorie food pictures: $M=5.3, S D=0.6$; low calorie food pictures: $M=5.1, S D=0.6$; ] were selected for the actual study. From the object Pictures 15 neutral items $(M=4.0, S D=0.3$; e.g. light bulb, staples, golf ball $)$ were selected [ratings did not significantly deviate from the neutral score $4 ; t(14)=0.54$, $P=0.60]$.

The use of pictures instead of real food is supported by prior research. First, Tiggemann and Kemps [57] showed that the visual modality was judged as most important in the mental imagery of food craving (visual modality $39.7 \%$, gustatory $30.6 \%$ and olfaction $15.8 \%$ ). Second, using fMRI, Simmons et al. [58] showed that food pictures activate gustatory areas for taste and reward. Finally, Kringelbach and Rolls [59] argue that the cells in OFC respond strongest to visual modality of food stimuli.

The pictures were projected as pop-out figures on a black canvas to minimize noise input [60]. Noise input is defined as all information not directly related to the relevant stimulus. Another advantage is that the combination of a pop-out figure on a black background in a dark environment (scanner bore) results in a presentation of the stimulus without environmental cues about the actual size of the presented stimulus. By using a black background, the 'naturalness' of the stimul is not disturbed by a perceived difference in stimulus size compared to the real object size.

\subsection{Design and experimental task}

This study used a 3 (picture types: high-caloric foods, low-caloric foods, neutral objects) $\times 2$ (deprivation status: satiated, $18 \mathrm{~h}$ food deprived) $\times 2$ (attention focus: object/food attended, bars attended) within-subjects design.

Following Pessoa et al. [47], in the experimental task participants were shown pictures of foods and neutral objects in the center of the screen with bars aside (Fig. 1). At the beginning of each block a word was shown that indicated the task of the participants. In the foods attended blocks, participants were shown the word "taste", prompting participants to judge the palatability of the presented foods (+: index finger, - : middle finger). The neutral objects attended blocks were preceded by the word "red" and participants were asked to indicate whether the presented objects were red (+: index finger, -: middle finger). In the bars attended blocks, the word "bars" was shown and participants had to indicate whether the bars were of similar orientations (+: index finger, - : middle finger). In the foods attended conditions participants were asked to respond to the question 'how do you like the taste of the food'. They were told that it was extremely important to vividly imagine the taste of the foods during the taste blocks, as if they were actually consuming the food. Participants were not informed about the difference in caloric content of the food pictures. Finally, participants were instructed that it was important to respond as accurately as possible.

\subsection{Stimulation protocol}

During the scanning session, the food, neutral object and bar-orientation conditions of the experimental task were presented in a blocked fashion. Each block ( $18 \mathrm{~s})$ consisted of an initial word instruction (taste, red or bars; $3000 \mathrm{~ms}$ ) and 5 stimulus trials ( $3000 \mathrm{~ms}$ each) with 5 low-caloric foods, 5 high-caloric foods, or 5 neutra objects. Each trial started with a $200 \mathrm{~ms}$ display of a food or object and two peripheral bars to the right and left at $6^{\circ}$ eccentricity. After this stimulus display, a white fixation cross was shown for $2800 \mathrm{~ms}$ (see Fig. 1) during which the participant could give her response. Each 18 s block was followed by a fixation block of $9 \mathrm{~s}$. Participants were explicitly instructed that fixation should be maintained throughout the experiment. The brief $200 \mathrm{~ms}$ display and the positioning of the stimuli within the centre of the visual field eliminated the occurrence of deliberate eye saccades [47].
For each of three runs, block-order was fully randomized and then manually checked for repetitions. For each separate participant, the order of the trials within each block was randomized. For the bar-orientation trials, 50\% of the bars were matches and 50\% were non-matches. Each high-caloric food, low-caloric food and neutral object was once displayed with matching bars and once with non-matching bars. Each block (high-caloric food and taste, low-caloric food and taste, neutral objects and red, high-caloric food and bars, low-caloric food and bars, neutral object and bars) was presented six times per run. Each run thus comprised 36 blocks separated by fixation periods, and lasted $17 \mathrm{~min}$. Each session consisted of three runs and two anatomical scans. The order of the runs was balanced across participants, but was kept constant over sessions within one participant. Similarly, the order of the response hand was balanced across participants and runs, but was kept constant over sessions within one participant. The order of the two scanning sessions (food deprived vs. satiated) was also balanced across participants.

\subsection{Procedure}

Each participant underwent two fMRI sessions (food deprived and satiated) which were scheduled at least one week apart. In the food deprived condition, participants were instructed not to consume any food or beverages (except water) $18 \mathrm{~h}$ prior to the imaging session. In the satiated condition, the participant was provided with a lunch containing $500 \mathrm{kcal}$, half an hour prior to the imaging session. All imaging sessions took place around lunch-time (i.e., between 1 and 3 PM). To ensure that the food deprivation worked, prior to each session hunger was assessed with $100 \mathrm{~mm}$ visual analogue scales (VAS; translated into Dutch by [61]). The VAS questionnaire also included ratings about more general physical sensations (e.g., headache, nausea, dizziness, anxiety) to see if the food deprivation had any effects on participants' general wellbeing. Prior to entering the scanner, the participant was trained on a practice task outside the scanner, which was similar to the experimental task inside the scanner. After completing the practice task, the participant entered the scanner and completed the experimental task. Each fMRI session lasted $75 \mathrm{~min}$. At the end of the two fMRI sessions, the participant completed an exit questionnaire, inquiring about their general experience with the fMRI experiment.

\section{6. fMRI data acquisition}

Images were acquired with a 3T Siemens Magnetom Allegra Head-only Scanner at the Maastricht Brain Imaging Centre (MBIC) using a birdcage volume coil. Gradient-echo planar imaging (EPI) volumes were acquired ( 50 slices, $\mathrm{TR}=3000 \mathrm{~ms}$ ). Imaging parameters were optimized to minimize susceptibility and distortion artifacts in OFC [62]. The relevant factors included oblique axial imaging with a negative (i.e. backward) tilt angle of $30^{\circ}$, minimizing voxel size $(2 \mathrm{~mm} \times 2 \mathrm{~mm} \times 2.5 \mathrm{~mm})$ in the plane of the imaging, a short echo time of $25 \mathrm{~ms}$, and a high imaging bandwidth $(2790 \mathrm{~Hz}$ over the field of view, echo spacing $=0.4 \mathrm{~ms})$. The voxel matrix size was $128 \times 104$ and the field of view (FoV) was $256 \mathrm{~mm} \times 208 \mathrm{~mm}$. Acquisition of functional images yielded 340 volumes per run. Two high-resolution wholebrain anatomical T1-weighted scans were acquired: an MDEFT [63](TR $=7.9 \mathrm{~ms}$, $\mathrm{TE}=2.4 \mathrm{~ms}$, flip angle $=15^{\circ}, 1 \mathrm{~mm} \times 1 \mathrm{~mm} \times 1 \mathrm{~mm}$ ) an optimized MPRAGE sequence $\left(\mathrm{TR}=2250 \mathrm{~ms}, \mathrm{TE}=2.6 \mathrm{~ms}\right.$, flip angle $\left.=9^{\circ}, 1 \mathrm{~mm} \times 1 \mathrm{~mm} \times 1 \mathrm{~mm}\right)$.

\section{7. fMRI data preprocessing}

All processing and analysis of the fMRI data was performed using Brainvoyager QX (v 1.9). The first two volumes of the T2* weighted functional images were discarded due to magnetic saturation effects. Preprocessing comprised slice scan timing correction (using sinc interpolation), motion correction (using a 3D rigid-body transformation of each volume to the first volume of each run and using trilinear/sinc interpolation) and high-pass filtering to remove low-frequency noise (up to three cycles in the single run time-course). Individual functional data were smoothed using a $6 \mathrm{~mm}$ full-width-at-half-maximum isotropic Gaussian Kernel.

For each participant, the four anatomical scans obtained throughout the experiment were averaged using a 3D rigid-body alignment to obtain a high-resolution and high contrast anatomical scan. The skull and cerebellum were removed by an automatic skull stripping procedure. Functional data were averaged for each participant per condition and aligned with the mean anatomical scan.

The mean anatomical scan and the functional data were then spatially normalized using Talairach transformation procedures [64]. For group analysis, the normalized individual functional data were averaged, accounting for both scan-toscan and participant-to-participant variability.

\subsection{Analysis}

Random effects (RFX) analysis of variance (ANOVA) was performed to determine the effect of picture type, food deprivation and attention focus on percentage BOLD signal change per voxel. All created $F$-maps were thresholded at a significance level of $P<0.001$ and a cluster size of 30 contiguous voxels (not corrected for multiple comparisons). Event related averaging (ERA) plots were created, visualizing the mean\% change in BOLD response in the significantly active regions of the resulting $F$-map. 


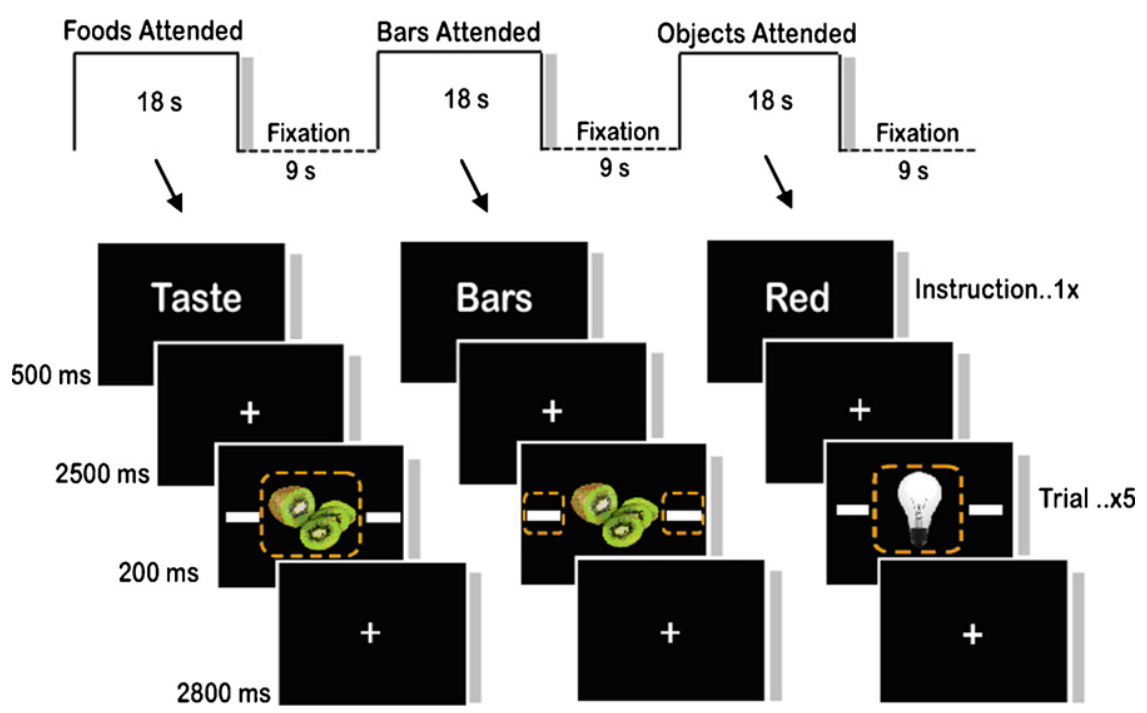

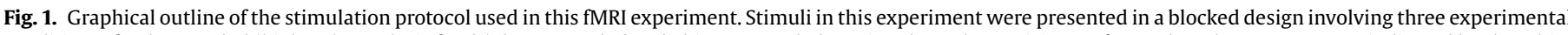

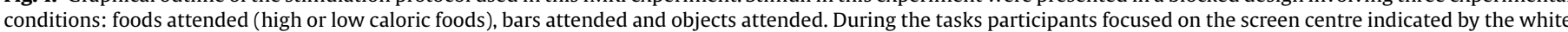

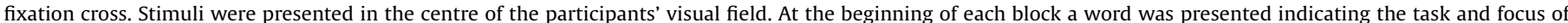

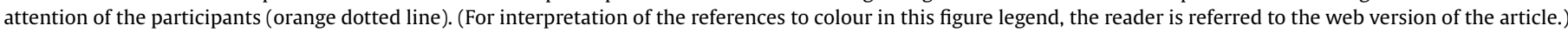

Functional Regions of interest (fROIs) were identified based on the hypothesis of current interest. The RFX Analysis showed that none of the voxels showed significant activity for the three-way interaction (picture type $\times$ food deprivation $\times$ attention focus). Therefore, analysis of the fMRI data is mainly based on the F-map of the two-way interaction: pictures $\times$ attention.

To test our hypotheses, a whole-brain statistical F-map was created of the RFX ANOVA F-test: picture type (low calorie food, high calorie food or neutral object) $\times$ attention focus (object/foods attended or bars attended). The resulting $F$ map revealed a network of significantly active brain regions. A brain region was indicated as fROI if significant BOLD response following the low and/or high calorie foods attended conditions, but not the neutral objects attended conditions. This allowed us to separate neutral object processing regions from food processing regions. The mean $F$-value of all voxels within each marked fROI is reported (Table 1) and anatomical localization was achieved using the Talairach coordinates corresponding to the center of these fROIs. In a second-level analysis, the average voxel beta values of all voxels within the identified fROIs (per condition for each participant individually, resulting in a total of 144 beta's ( 12 participants $\times 12$ conditions) per fROI) were extracted and submitted to a repeated measure ANOVA in SPSS. This allowed us to test the effects of calorie content and food deprivation on BOLD activity within each fROI, especially the amygdala and OFC.

The third hypothesis concerned the functional distinction between the amygdala and OFC due to differences in attentional focus. To test the first part of this hypothesis (explicit food evaluation is necessary for significant OFC activity), fROIs had to be identified that responded significantly to the food conditions depending on attention focus. To achieve this goal the same RFX ANOVA F-map (pictures $\times$ attention focus) and second-level analysis were performed as for the first hypothesis. Finally, to test the second part of the third hypothesis (automatic incentive evaluation processing in the amygdala, independent of attention focus), a conjunction effects analysis was performed searching for brain activation common to all food conditions (over foods attended and bars attended conditions) versus all neutral object conditions (over neutral objects attended and bars attended conditions) in both satiated and food deprived conditions. Therefore, the conjunction test performed was food (satiated) versus neutral (satiated) AND food (deprived) versus neutral (deprived). The voxel beta values of fROIs were extracted from each participant individually and submitted to a repeated measure ANOVA in SPSS.

Unfortunately the RFX ANOVA F-test we used to analyze our fMRI data did not show any significant activity following the three-way interaction (picture type $\times$ food deprivation $\times$ attention focus). Lowering the cluster size threshold or decreasing the voxel-wise significance criterion did not change this null finding. However, because effects caused by calorie content or food deprivation may be very small, our RFX analyses may have been to restrictive (type II error). Therefore in addition we applied a less strict conjunction effects analyses. A conjunction effects analysis allows for the search of significant effects following more than one $t$-contrast within a single voxel. Following our hypothesis of the effects of food deprivation and calorie content on reward processing, we combined two $t$-contrasts. First we tested for significant activation common to all food conditions $(+)$ versus all neutral objects $(-)$ in both food deprived and satiated attended conditions. Then we combined this $t$-contrast with a second that tested for significant interactions between food deprivation and energy content [(high calorie foods attended satiated + low calorie foods hungry) $<$ (high calorie foods attended hungry + low calorie foods satiated)]. The effects revealed by this conjunction analysis were indeed smaller compared to those of the attention manipulation. Therefore the conjunction effects $t$-maps were thresholded at a less strict voxel-wise significance criterion of $P<0.05$ and a cluster size threshold of 10 contiguous voxels (no correction for mul-

Table 1

Results whole-brain RFX ANOVA. F-test: Picture (neutral object vs. low and high calorie foods) $\times$ attention focus (neutral object/food attended vs. bars attended).

\begin{tabular}{|c|c|c|c|c|c|}
\hline Number & Functional region of interest (fROI) & $L / R$ & Talairach coordinates $(x, y, z)$ & BA & $F$-score \\
\hline 1 & Fusiform gyrus & B & $-46,-54,-13$ & 37 & 30.52 \\
\hline \multirow[t]{3}{*}{2} & Basal ganglia & & & & \\
\hline & a. Thalamus & B & $-12,-16,12$ & - & 26.84 \\
\hline & b. Ventral striatum & B & $-15,-1,4$ & - & 28.31 \\
\hline 3 & Amygdala & $\mathrm{L}$ & $-13,-8,-5$ & 34 & 34.15 \\
\hline 4 & Premotor cortex & $\mathrm{L}$ & $-3,1,54$ & 6 & 21.06 \\
\hline 5 & Inferior frontal gyrus, pars opercularis & $\mathrm{L}$ & $-46,3,31$ & 44 & 20.77 \\
\hline 6 & Anterior cingulate cortex & $\mathrm{L}$ & $-1,10,36$ & 24 & 21.13 \\
\hline 7 & Insula/frontal operculum & B & $-32,15,8$ & 47 & 32.33 \\
\hline 8 & Medial orbitofrontal cortex & $\mathrm{L}$ & $-24,29,-4$ & 11 & 31.47 \\
\hline 9 & Ventrolateral prefrontal cortex & $\mathrm{L}$ & $-44,36,23$ & 45 & 22.55 \\
\hline 10 & Dorsolateral prefrontal cortex & $\mathrm{L}$ & $-28,42,36$ & $46 / 9$ & 27.76 \\
\hline
\end{tabular}

Note: $\mathrm{L}=$ left; $\mathrm{R}=$ right; $\mathrm{B}=$ bilateral; $\mathrm{BA}=$ Brodmann area. 
Table 2

Results conjunction effects analyses.

\begin{tabular}{|c|c|c|c|c|c|c|}
\hline Number & Functional region of interest & $L / R$ & Talairach coordinates & BA & $t$-value & $p$-value \\
\hline 1 & Posterior cingulate cortex & $\mathrm{L}$ & $-6,-22,34$ & 23 & 2.73 & 0.004 \\
\hline 2 & Lateral orbitofrontal cortex & $\mathrm{L}$ & $-40,36,0$ & 11 & 2.51 & 0.009 \\
\hline 3 & Insula & $\mathrm{R}$ & $35,3,4$ & 47 & 2.57 & 0.003 \\
\hline 4 & Medial orbitofrontal cortex & $\mathrm{L}$ & $-23,34,0$ & 11 & 2.33 & 0.015 \\
\hline 5 & Medial orbitofrontal cortex & $\mathrm{R}$ & $30,28,0$ & 11 & 2.44 & 0.008 \\
\hline 6 & Putamen & $\mathrm{L}$ & $-24,-8,-4$ & - & 2.45 & 0.008 \\
\hline 7 & Fusiform gyrus & $\mathrm{L}$ & $-18,-49,-11$ & 37 & 2.36 & 0.026 \\
\hline
\end{tabular}

Note: $\mathrm{L}=$ left; $\mathrm{R}=$ right; $\mathrm{BA}=$ Brodmann area.

tiple comparison). From each $t$-map fROIs were identified. The average $t$-values of these fROIs within each map are reported (Table 2) and anatomical localization was achieved using the Talairach coordinates corresponding to the center of these fROIs. The average beta weights were again extracted and submitted to a repeated measure ANOVA in SPSS.

\section{Results}

\subsection{Behavioural results}

Confirming that our food deprivation worked, a paired-samples $t$-test of the subjective hunger ratings revealed a significant difference between the hunger and satiety condition [deprived: $M=84.50, S D=7.64$; satiated: $M=12.83, S D=6.93 ; t(11)=20.74$, $P<0.001]$. There were no differences between the two conditions on subjective ratings of headache, nausea or anxiety [all $p s \geq .05$, largest $t(11)=1.56]$, but participants did score higher on dizziness after food deprivation than when satiated [deprived: $M=15.94$, $S D=13.42$; satiated: $M=3.37, S D=2.42 ; t(11)=2.73, P<0.05]$.

As a measure of attendance we additionally calculated the average correct response rate of the participants during all bars attended conditions. Results showed that participants responded correctly on $96.01 \%$ of the trials.

\section{2. fMRI results}

\subsubsection{Hypothesis I: Calorie content and reward}

The whole-brain analysis of the pictures (low calorie foods, high calorie foods vs. neutral objects) $\times$ attention focus (neutral object/food attended vs. bars attended), revealed a network of areas showing significant activity. Functional regions of interest (fROIs) were identified, in which significant BOLD response following the low and high calorie foods attended conditions. Together the resulting fROIs outlined a food processing network, including the left amygdala and left medial OFC (MOFC) as shown in Fig. 2.

The second-level analysis of the beta weights revealed that there was no significant difference in BOLD activity between the high and low calorie foods in both the food deprived and satiated attended conditions in the left amygdala, both when participants where food deprived [high calorie foods: $M=0.12$, $S D=0.10$; low calorie foods: $M=0.12, S D=0.09 ; t(11)=0.08, P=0.94]$ or satiated [high calorie foods: $M=0.12, S D=0.17$; low calorie foods: $M=0.16, S D=0.14 ; t(11)=0.17, P=0.97]$. A similar null effect for the calorie content was found in left OFC for both the food deprived [high calorie foods: $M=0.20, S D=0.12$; low calorie foods: $M=0.23, S D=0.12 ; t(11)=0.34, P=0.74$ ] and satiated con-
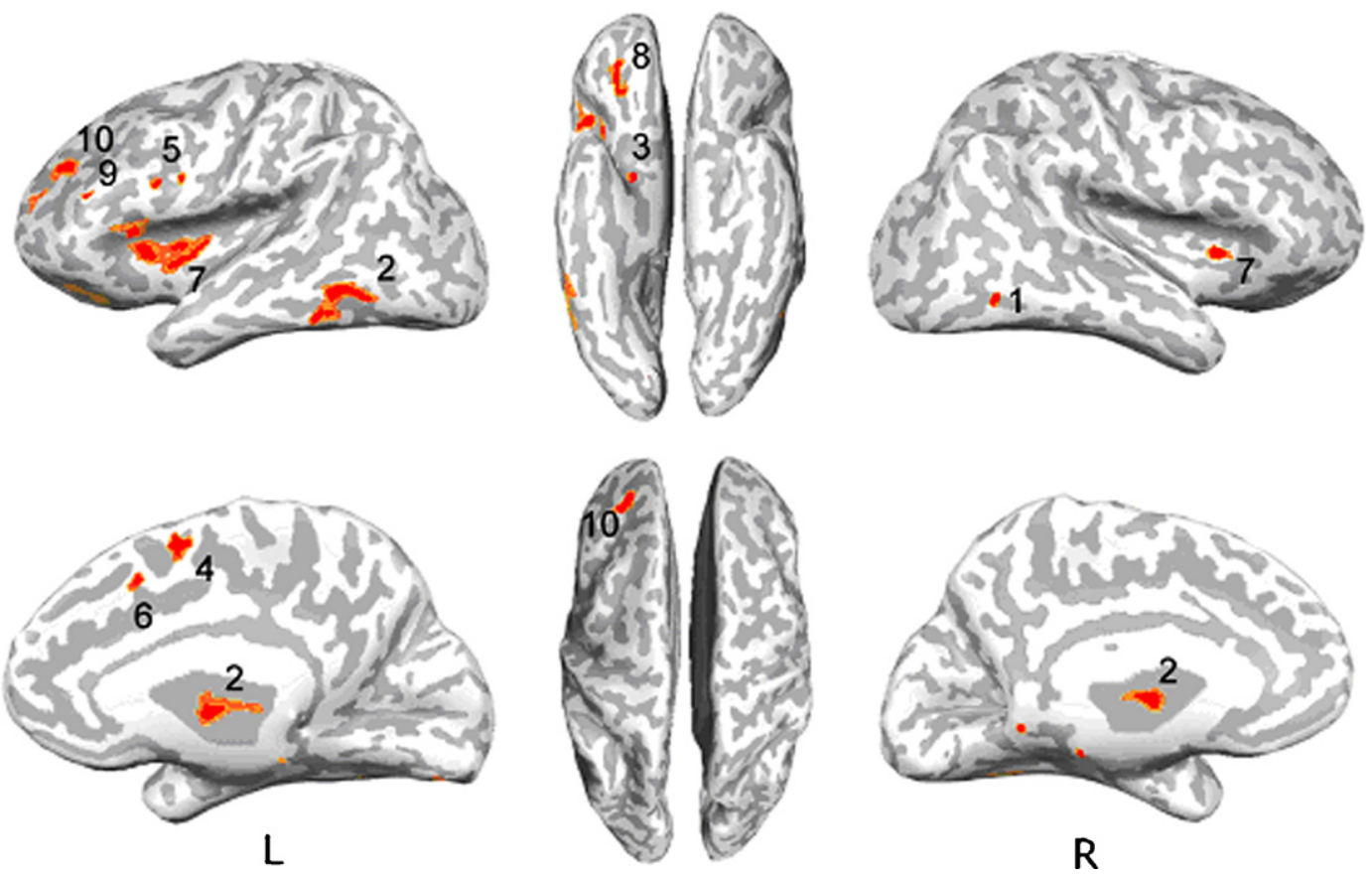

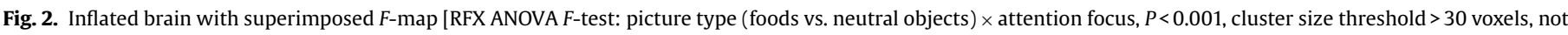

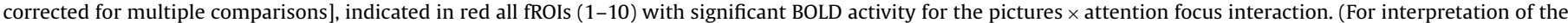
references to colour in this figure legend, the reader is referred to the web version of the article.) 


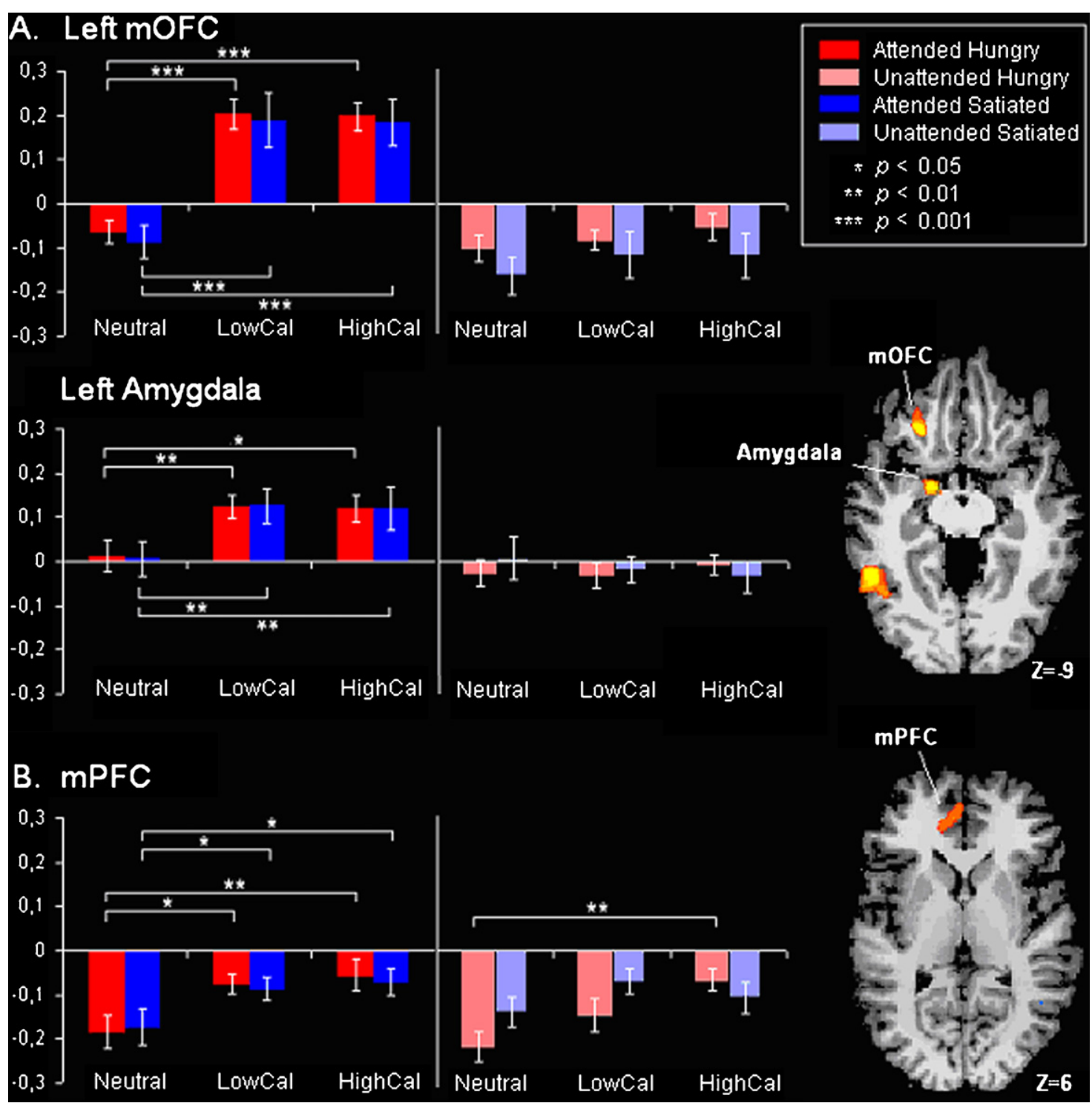

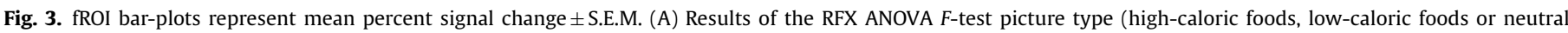

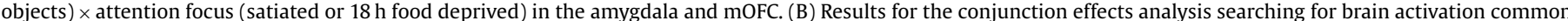
to all food conditions independent of attention, in both satiated and food deprived conditions.

dition [high calorie foods: $M=0.18, S D=0.19$; low calorie foods: $M=0.19, S D=0.21 ; t(11)=0.15, P=0.89]$. These non-significant effects are clearly visualized in Fig. 3A. Further examination of difference in BOLD response between the high and low calorie food conditions in the food deprived and satiated condition did not reveal any significant effect in any of the remaining fROIs.

\subsubsection{Hypotheses II: Food deprivation and reward}

The second-level analysis following the RFX ANOVA F-test: picture type (low calorie food, high calorie food or neutral object) $\times$ attention focus revealed that there was no significant difference in BOLD activity between the high and low calorie foods in both the food deprived and satiated attended conditions in the left amygdala or mOFC [Fig. 3A]. The second-level Repeated Measures ANOVA analyses of the beta values of the remaining fROIs did not show a significant effect of food deprivation on the (attended) food conditions either.

\subsubsection{Hypotheses III: Attention focus}

The whole-brain statistical F-map of the RFX ANOVA F-test picture type (neutral object vs. foods averaged) $\times$ attention focus (neutral object/food attended vs. bars attended) showed a stronger BOLD response in the left mOFC in the attended foods averaged conditions (Fig. 3A) as compared to the bars attended or object attended conditions. This result confirms our hypothesis that explicit evaluation of foods is necessary for OFC activity Furthermore, other regions believed to be critically involved in food processing were also strongly modulated by attention focus (e.g. ventral striatum, premotor cortex, anterior cingulate cortex, insula/frontal operculum, ventrolateral prefrontal cortex and dorsolateral prefrontal cortex; Fig. 4).

Conjunction effects analysis for brain activation common to all food conditions (both foods attended and bars attended conditions) versus the neutral object conditions (both objects attended and bars attended conditions) revealed no significant effect in the amygdala (Fig. 3A). As a result, the hypothesis that amygdala activity is 


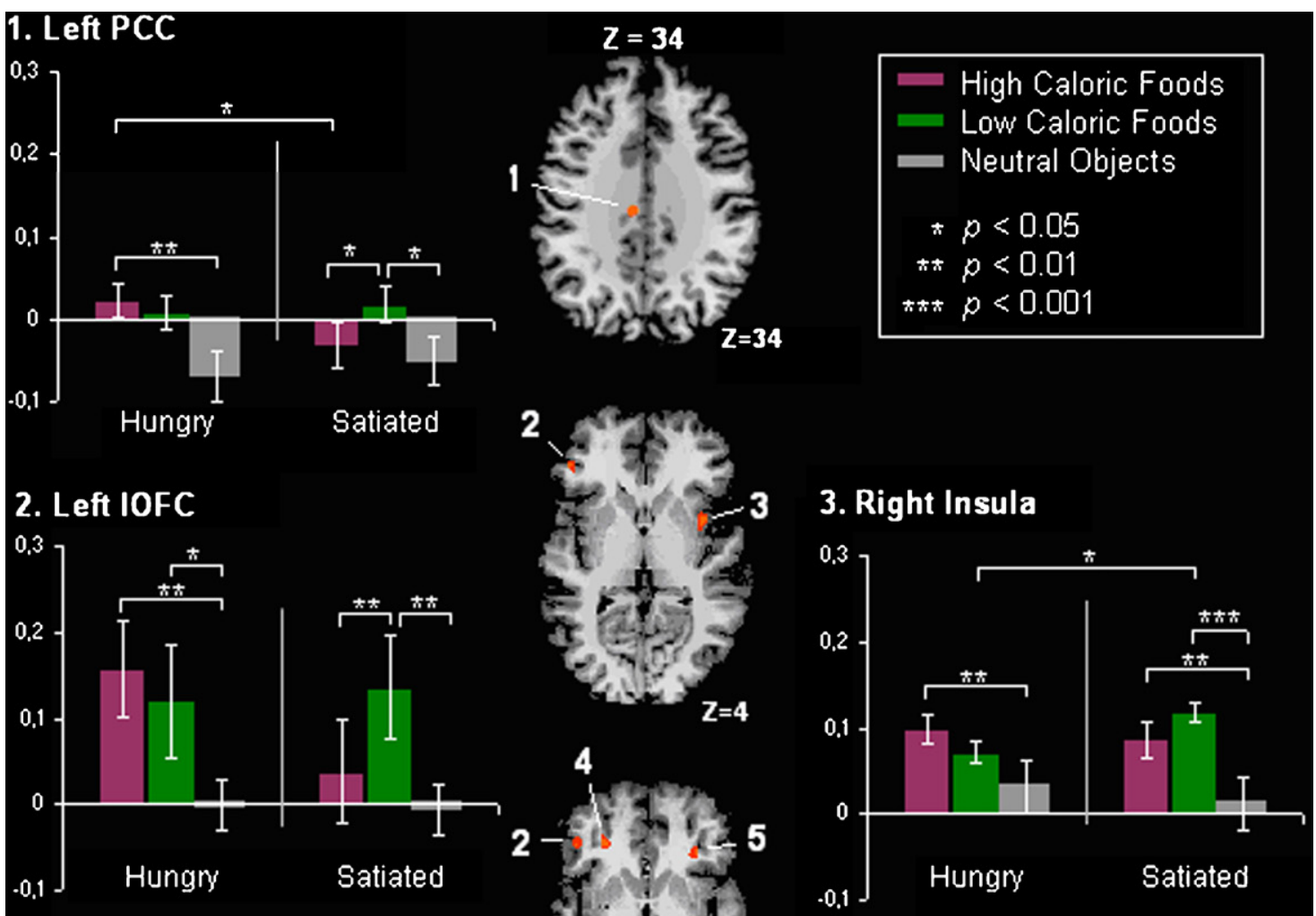

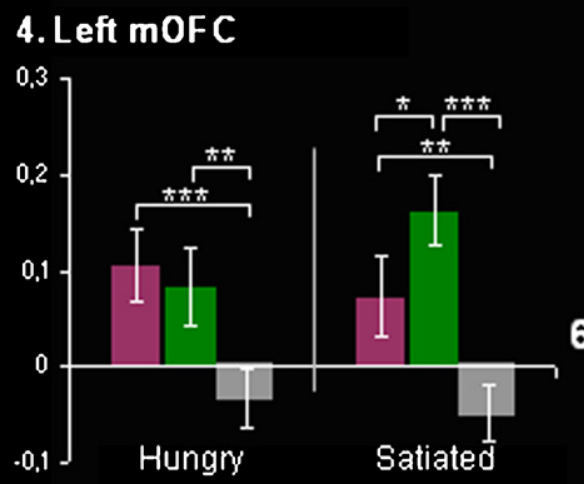

6. Left Caudate Putamen

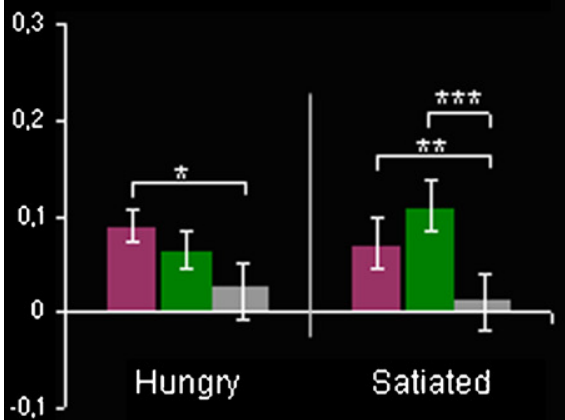

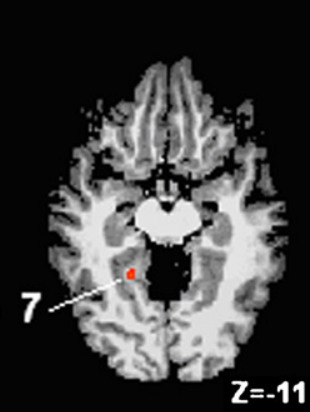

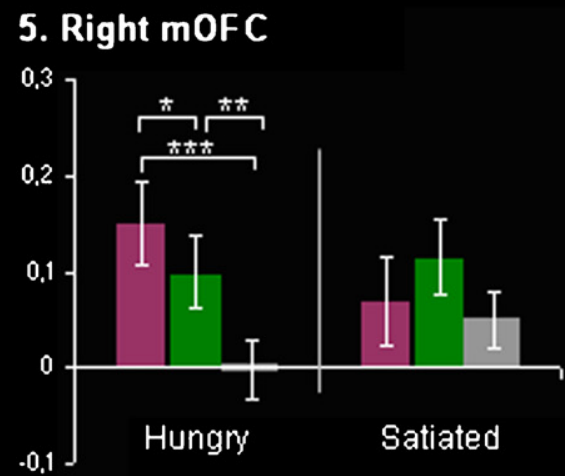

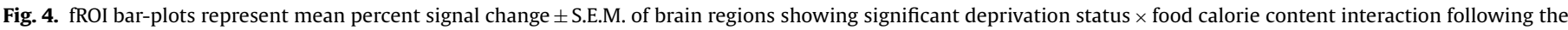
additional conjunction effects analysis.

independent of focus of attention was left unconfirmed. However, a significantly reduced inhibition of left medial prefrontal cortex ( $\mathrm{MPFC}$ ) activity was revealed following presentation the high calorie food conditions, independent of focus of attention focus (Fig. 3B).
2.2.4. Additional conjunction effects analysis

The whole-brain analysis of the effects of food deprivation in conjunction with the interaction with calorie revealed a network of significantly active areas, including the hypothesized OFC. 
Other identified fROIS included the posterior cingulate cortex (PCC), insula, caudate putamen and fusiform gyrus (Table 2, Fig. 4). Results showed that overall activity in these fROIs was strongest for the low calorie food stimuli in the satiated conditions. Hunger increased the activity for high calorie stimuli these fROIs especially in the fusiform gyrus, right mOFC, right insula, left caudate putamen and PCC. This finding suggest that food deprivation indeed interacts with the caloric content of food stimuli and confirms our hypothesis that high calorie food stimuli become more rewarding when hungry.

\section{Discussion}

In this fMRI study, we examined the modulating effects of calorie content, hunger and attention focus on food reward processes in the human brain. The data show that attending to and evaluating food pictures strongly activates a large network of left-sided brain regions, including the fusiform gyrus, ventral striatum, amygdala, bilateral insula/frontal operculum, ACC, premotor area, dIPFC and mOFC. The involvement of these brain areas in the explicit processing of food cues is in accordance with previous findings [58]. As anticipated, this 'food processing network' was highly lateralized to the left-hemisphere. Numerous neuroimaging studies, using a visual working memory task and right-handed participants, show a clear lateralization of brain activity in the left-hemisphere [65-68]. The highly significant BOLD activity in the insula/frontal operculum (also referred to as the primary gustatory cortex) confirmed that the participants successfully imagined the taste of the foods, as they were explicitly instructed to do.

Although self-reports showed a successful manipulation of food deprivation on subjective ratings of hunger, the initially applied RFX ANOVA test revealed no effect of food deprivation or calorie content on reward evaluation processing in the amygdala or OFC. This suggested that hunger did not modulate the explicit reward evaluation of the high and low calorie food cues, which was a quite unexpected finding. Interestingly, a less strict conjunction analysis did reveal the hypothesized results, with significant activity in the lateral and medial OFC, PCC, caudate putamen, fusiform gyrus and the insula. Activity in these areas was strongest for the low calorie foods when the healthy females were satiated and stronger for the high calorie foods when they were hungry. The involvement of these regions in normal and abnormal reward processing is supported by several neuroimaging studies [69-74].

The finding of a modulation by hunger and calorie content in the fusiform gyrus is in accordance with the model proposed by Murray and Izquierdo [16], who suggest that there are two rewardprocessing pathways to the OFC. The first pathway concerns the reward evaluation interactions between the OFC and amygdala. The second route involves interactions between the OFC and the inferotemporal cortex, which comprises the fusiform gyrus. Murray and Izquirdo propose that this pathway allows for visual cues to elicit the predicted values of objects and is important in determining rules for future actions.

Another structure modulated by the hunger and calorie content manipulation was the right insula. Insular activity has previously been shown to be correlated with subjective cue-induced drug cravings [75]. It has also been shown that right insula activity is associated with relapse to alcohol use [76]. Furthermore, damage to the insula has been reported to disrupt addiction to smoking [77]. It is proposed that the insula is especially involved in the conscious craving of rewards through its role in the representation of bodily states. The right insular activity found in this study might therefore reflect the participants' conscious craving or 'wanting' of the presented foods.
The finding that high calorie foods become extra rewarding when hungry has an important clinical implication. We previously indicated that energy restriction is one of the most applied strategies in the treatment of obesity [42]. The finding of an increased reward value of energy-dense foods when hungry gives a possible reason why dietary restriction may be difficult. Diets limited in their energy content but with good satiating properties, for example high-protein diets [78], could increase success. Furthermore, our results indicate that healthy females prefer low calorie foods when satiated. It can be hypothesized that obese people, in contrast to healthy-weight females, have increased reward processing following the presentation of high calorie foods compared to low calorie foods when satiated. This could explain part of the mechanism underlying their abnormal eating behaviour, but of course needs to be studied first.

To test the hypothesis whether an explicit evaluation of taste is necessary for OFC activity, but not amygdalar activity, we manipulated the attention focus of the participants. Results revealed a highly significant effect of attention focus on the BOLD response in several regions including the $\mathrm{mOFC}$, but also the amygdala. These results suggest that explicit subjective evaluation of foods is necessary to elicit both amygdalar and mOFC activity. There was no functional dissociation between the amygdala and OFC based on differences in attention focus. The present attention effect on mOFC processing is in line with previous findings suggesting that mOFC is involved in the 'conscious' experience of reward $[9,10,40]$. However, the finding that explicit evaluation is also necessary for amygdalar processes was unpredicted. Our results suggest that amygdalar reward processing depends on attention focus and does not occur automatically. It has been suggested that studies revealing attention dependent amygdalar processing use very demanding tasks exhausting all processing capacity [79]. If this is true, none of the significantly active regions should reveal an attention independent activation. However, in our study attention independent processing was found in the MPFC following presentation of high calorie foods when participants were hungry. MPFC activity has frequently been reported in fMRI studies $[33,80]$. However, the precise function of this region remains unclear. Previous research indicates that mPFC might be involved in self-monitoring [81] and the coordination of external versus internally generated information [82]. The attention independent activity in MPFC found in this study might represent the hungry participants' "awareness" of biologically relevant and salient high calorie foods also in the bars attended conditions. This suggestion is supported by reports in the exit questionnaires, in which participants stated that they were conscious of the presences of foods in the bars attended conditions. Therefore, our results are in line with the hypothesized role of self-monitoring and information coordination by $\mathrm{mPFC}$.

Of special interest are the strong effects of the attention manipulation compared to the smaller effects of hunger and calorie content. This finding leads to several interesting hypotheses. For example, the strong effect of the attention manipulation may have been the result of our task instructions. In the food attended blocks, participants were explicitly instructed to vividly imagine the taste of the presented food pictures to evaluate their palatability. Research indicates that explicit memory processes are highly task-dependent [83] and that changes in task instructions modulate representations in working memory [84]. In this study, to correctly perform the taste task ("How do you like the taste of this food?"), it was not necessary to actively retrieve information about the physical state of the body. This may have resulted in the weaker hunger and calorie content modulation effects, as participants could answer this question without considering their physical state. Asking participants a motivational question, for example: "How much would you like to eat this food right now?" might have yielded 
stronger effects. Furthermore, in this study the satiated participants were explicitly instructed to evaluate the foods, while in 'real world' situations people probably do not evaluate foods when satiated, unless they are explicitly instructed to do so. Presenting hungry and satiated participants with a passive fMRI paradigm (no explicit evaluation of food palatability) is necessary to test this post hoc explanation, as it allows participants to evaluate the foods but does not make it a task requirement. Furthermore it would be interesting to test whether obese people might frequently imagine or evaluate the palatability of foods, even when they are satiated. This would then elicit reward processes that might be experienced as craving, overruling a natural inhibition mechanism of eating behaviour.

In conclusion, robust left-hemisphere explicit food processing activity was found in the present study. Hunger and calorie content influenced reward processing in the lateral and medial OFC, cingulate cortex, caudate putamen, insula and fusiform gyrus. Confirming our hypothesis, satiated healthy females show a stronger BOLD response in these reward-processing areas following the presentation of low calorie foods, whereas hungry healthy females showed a stronger BOLD response in these areas when presented with high calorie foods. This increase in activity is likely to represent an increase in reward value of the high calorie foods and may explain why treatments of obesity energy restricting diets are often unsuccessful. Our study highlighted the importance of attention focus in food reward processing, showing strong BOLD activity in the amygdala and mOFC when participants attended the foods, but no activity at all if the task did not require the evaluation of the foods. Attention independent processing was found in the MPFC following the presentation of high calorie foods when participants were hungry, an area proposed to be involved in linking reward to actions. These results suggest that a further investigation of attentional processes on the modulation of reward processing might be especially informative in determining the neural substrates of healthy and pathological eating behaviour.

\section{Acknowledgement}

We thank Armin Heinecke of Brain Innovation B.V. for his help and assistance with the analyses of the fMRI data and Sven Gijsen for his technical support with the MRI scanner. Grateful acknowledgement for proofreading goes to Danielle Tisserand. Finally we would like to thank an anonymous reviewer for suggesting a less stringent analysis method.

\section{References}

[1] Davis C, Patte K, Levitan R, Reid C, Tweed S, Curtis C. From motivation to behaviour: a model of reward sensitivity, overeating, and food preferences in the risk profile for obesity. Appetite 2007;48:12-9.

[2] Epstein LH, Leddy JJ, Temple JL, Faith MS. Food reinforcement and eating: a multilevel analysis. Psychol Bull 2007;133:884-906.

[3] Robinson TE, Berridge KC. Addiction. Annu Rev Psychol 2003;54:25-53.

[4] Kelley AE, Berridge KC. The neuroscience of natural rewards: relevance to addictive drugs. J Neurosci 2002;22:3306-11.

[5] Rolls ET. Sensory processing in the brain related to the control of food intake. Proc Nutr Soc 2007;66:96-112.

[6] Wang GJ, Yang J, Volkow ND, Telang F, Ma Y, Zhu W, et al. Gastric stimulation in obese subjects activates the hippocampus and other regions involved in brain reward circuitry. Proc Natl Acad Sci U S A 2006;103:15641-5.

[7] Arana FS, Parkinson JA, Hinton E, Holland AJ, Owen AM, Roberts AC. Dissociable contributions of the human amygdala and orbitofrontal cortex to incentive motivation and goal selection. J Neurosci 2003;23:9632-8.

[8] De Araujo IE, Rolls ET. Representation in the human brain of food texture and oral fat. J Neurosci 2004;24:3086-93.

[9] Gottfried JA, O'Doherty J, Dolan RJ. Encoding predictive reward value in human amygdala and orbitofrontal cortex. Science 2003;301:1104-7.

[10] Kringelbach ML, O’Doherty J, Rolls ET, Andrews C. Activation of the human orbitofrontal cortex to a liquid food stimulus is correlated with its subjective pleasantness. Cereb Cortex 2003;13:1064-71.
[11] O'Doherty J, Rolls ET, Francis S, Bowtell R, McGlone F, Kobal G, et al. Sensoryspecific satiety-related olfactory activation of the human orbitofrontal cortex. Neuroreport 2000;11:893-7.

[12] O'Doherty J, Rolls ET, Francis S, Bowtell R, McGlone F. Representation of pleasant and aversive taste in the human brain. J Neurophysiol 2001;85:1315-21.

[13] Small DM, Veldhuizen MG, Felsted J, Mak YE, McGlone F. Separable substrates for anticipatory and consummatory food chemosensation. Neuron 2008;57:786-97.

[14] Ghashghaei HT, Barbas H. Pathways for emotion: interactions of prefrontal and anterior temporal pathways in the amygdala of the rhesus monkey. Neuroscience 2002;115:1261-79.

[15] Goldstein RZ, Volkow ND. Drug addiction and its underlying neurobiological basis: neuroimaging evidence for the involvement of the frontal cortex. Am J Psychiatry 2002;159:1642-52.

[16] Murray EA, Izquierdo A. Orbitofrontal cortex and amygdala contributions to affect and action in primates. Ann N Y Acad Sci 2007;1121:273-96.

[17] Volkow ND, Wise RA. How can drug addiction help us understand obesity? Nat Neurosci 2005;8:555-60.

[18] Wang GJ, Volkow ND, Telang F, Jayne M, Ma J, Rao M, et al. Exposure to appetitive food stimuli markedly activates the human brain. Neuroimage 2004;21: 1790-7.

[19] Volkow ND, Wang GJ, Telang F, Fowler JS, Logan J, Childress AR, et al. Dopamine increases in striatum do not elicit craving in cocaine abusers unless they are coupled with cocaine cues. Neuroimage 2008;39:1266-73.

[20] Volkow ND, Wang GJ, Fowler JS, Logan J, Gatley SJ, Hitzemann R, et al. Decreased striatal dopaminergic responsiveness in detoxified cocaine-dependent subjects. Nature 1997;386:830-3.

[21] Volkow ND, Wang GJ, Telang F, Fowler JS, Thanos PK, Logan J, et al. Low dopamine striatal D2 receptors are associated with prefrontal metabolism in obese subjects: possible contributing factors. Neuroimage 2008;42:1537-43.

[22] Wang GJ, Volkow ND, Logan J, Pappas NR, Wong CT, Zhu W, et al. Brain dopamine and obesity. Lancet 2001;357:354-7.

[23] Kringelbach ML. Food for thought: hedonic experience beyond homeostasis in the human brain. Neuroscience 2004;126:807-19.

[24] Weiskopf N, Hutton C, Josephs O, Deichmann R. Optimal EPI parameters for reduction of susceptibility-induced BOLD sensitivity losses: a whole-brain analysis at $3 \mathrm{~T}$ and $1.5 \mathrm{~T}$. Neuroimage 2006;33:493-504.

[25] Drewnowski A, Maillot M, Darmon N. Testing nutrient profile models in relation to energy density and energy cost. Eur J Clin Nutr 2008.

[26] MacArthur RH, Pianka E. An optimal use of a patchy environment. Am Nat 1966;100:603-9.

[27] Green SM, Wales JK, Lawton CL, Blundell JE. Comparison of high-fat and highcarbohydrate foods in a meal or snack on short-term fat and energy intakes in obese women. Br J Nutr 2000;84:521-30.

[28] Prentice AM. Manipulation of dietary fat and energy density and subsequent effects on substrate flux and food intake. Am J Clin Nutr 1998;67: 535S-41S.

[29] Rolls BJ. The role of energy density in the over consumption of fat. J Nutr 2000;130:268S-71S.

[30] Johnson J, Vickers Z. Factors influencing sensory-specific satiety. Appetite 1992;19:15-31.

[31] Yeomans MR, Lee MD, Gray RW, French SJ. Effects of test-meal palatability on compensatory eating following disguised fat and carbohydrate preloads. Int J Obes Relat Metab Disord 2001;25:1215-24.

[32] Rolls BJ, Laster LJ, Summerfelt A. Hunger and food intake following consumption of low-calorie foods. Appetite 1989;13:115-27.

[33] Killgore WD, Young AD, Femia LA, Bogorodzki P, Rogowska J, Yurgelun-Todd DA. Cortical limbic activation during viewing of high-versus low-calorie foods. Neuroimage 2003;19:1381-94.

[34] Berridge KC. Food reward: brain substrates of wanting and liking. Neurosci Biobehav Rev 1996;20:1-25.

[35] Berridge KC. Motivation concepts in behavioral neuroscience. Physiol Behav 2004;81:179-209.

[36] Robinson TE, Berridge KC. The psychology and neurobiology of addiction: an incentive-sensitization view. Addiction 2000;95(Suppl. 2):S91-117.

[37] Cabanac M:. Q Rev Biol 1979;54:1-29.

[38] Padoa-Schioppa C, Assad JA. Neurons in the orbitofrontal cortex encode economic value. Nature 2006;441:223-6.

[39] Rolls ET, Murzi E, Yaxley S, Thorpe SJ, Simpson SJ. Sensory-specific satiety. foodspecific reduction in responsiveness of ventral forebrain neurons after feeding in the monkey. Brain Res 1986;368:79-86.

[40] Small DM, Zatorre RJ, Dagher A, Evans AC, Jones-Gotman M. Changes in brain activity related to eating chocolate: from pleasure to aversion. Brain $2001 ; 124: 1720-33$

[41] Gilhooly CH, Das SK, Golden JK, McCrory MA, Dallal GE, Saltzman E, et al. Food cravings and energy regulation: the characteristics of craved foods and their relationship with eating behaviors and weight change during 6 months of dietary energy restriction. Int J Obes (Lond) 2007;31:1849-58.

[42] Epstein LH, Myers MD, Raynor HA, Saelens BE. Treatment of pediatric obesity. Pediatrics 1998;101:554-70.

[43] Jeffery RW, Drewnowski A, Epstein LH, Stunkard AJ, Wilson GT, Wing RR, et al. Long-term maintenance of weight loss: current status. Health Psychol 2000;19:5-16.

[44] Bishop SJ, Duncan J, Lawrence AD. State anxiety modulation of the amygdala response to unattended threat-related stimuli. J Neurosci 2004;24:10364-8. 
[45] Rauch AV, Ohrmann P, Bauer J, Kugel H, Engelien A, Arolt V, et al. Cognitive coping style modulates neural responses to emotional faces in healthy humans: a 3-T FMRI study. Cereb Cortex 2007;17:2526-35.

[46] Kensinger EA, Schacter DL. Processing emotional pictures and words: effects of valence and arousal. Cogn Affect Behav Neurosci 2006;6:110-26.

[47] Pessoa L, Padmala S, Morland T. Fate of unattended fearful faces in the amygdala is determined by both attentional resources and cognitive modulation. Neuroimage 2005;28:249-55.

[48] Vuilleumier P, Richardson MP, Armony JL, Driver J, Dolan RJ. Distant influences of amygdala lesion on visual cortical activation during emotional face processing. Nat Neurosci 2004;7:1271-8.

[49] Kringelbach ML. The human orbitofrontal cortex: linking reward to hedonic experience. Nat Rev Neurosci 2005;6:691-702.

[50] LaBar KS, Gitelman DR, Parrish TB, Kim YH, Nobre AC, Mesulam MM. Hunger selectively modulates corticolimbic activation to food stimuli in humans. Behav Neurosci 2001;115:493-500.

[51] Tataranni PA, Gautier JF, Chen K, Uecker A, Bandy D, Salbe AD, et al. Neuroanatomical correlates of hunger and satiation in humans using positron emission tomography. Proc Natl Acad Sci U S A 1999;96:4569-74.

[52] Herman CP, Polivy J, Pliner P, Threlkeld J, Munic D. Distractibility in dieters and nondieters: an alternative view of "externality". J Pers Soc Psychol 1978; $36: 536-48$

[53] Bryant M, Truesdale KP, Dye L. Modest changes in dietary intake across the menstrual cycle: implications for food intake research. Br J Nutr 2006;96:888-94.

[54] Goldzieher JW. Are low-dose oral contraceptives safer and better? Am J Obstet Gynecol 1994;171:587-90.

[55] Carver CS, White TL. Behavioral inhibition, behavioral activation, and affective responses to impending reward and punishment: the BIS/BAS Scales. J Pers Soc Psychol 1994;67:319-33.

[56] Spinella M. Normative data and a short form of the Barratt Impulsiveness Scale. Int J Neurosci 2007;117:359-68.

[57] Tiggemann M, Kemps E. The phenomenology of food cravings: the role of mental imagery. Appetite 2005;45:305-13.

[58] Simmons WK, Martin A, Barsalou LW. Pictures of appetizing foods activate gustatory cortices for taste and reward. Cereb Cortex 2005;15:1602-8.

[59] Kringelbach ML, Rolls ET. The functional neuroanatomy of the human orbitofrontal cortex: evidence from neuroimaging and neuropsychology. Prog Neurobiol 2004;72:341-72.

[60] Rainer G, Augath M, Trinath T, Logothetis NK. Nonmonotonic noise tuning of BOLD fMRI signal to natural images in the visual cortex of the anesthetized monkey. Curr Biol 2001;11:846-54.

[61] Friedman MI, Ulrich P, Mattes RD. A figurative measure of subjective hunger sensations. Appetite 1999;32:395-404.

[62] Deichmann R, Gottfried JA, Hutton C, Turner R. Optimized EPI for fMRI studies of the orbitofrontal cortex. Neuroimage 2003;19:430-41.

[63] Deichmann R. Optimized RF excitation for anatomical brain imaging of the occipital lobe using the 3D MDEFT sequence and a surface transmit coil. Magn Reson Med 2005;53:1212-6.

[64] Talairach J, Tournoux P. Co-planar stereotaxic atlas of the human brain. New York; 1988

[65] Cabeza R, Nyberg L. Imaging cognition II: an empirical review of 275 PET and fMRI studies. J Cogn Neurosci 2000;12:1-47.
[66] Herrington JD, Mohanty A, Koven NS, Fisher JE, Stewart JL, Banich MT, et al. Emotion-modulated performance and activity in left dorsolateral prefrontal cortex. Emotion 2005;5:200-7.

[67] Staresina BP, Davachi L. Differential encoding mechanisms for subsequent associative recognition and free recall. J Neurosci 2006;26: 9162-72.

[68] Volle E, Pochon JB, Lehericy S, Pillon B, Dubois B, Levy R. Specific cerebral networks for maintenance and response organization within working memory as evidenced by the 'double delay/double response' paradigm. Cereb Cortex 2005;15:1064-74.

[69] Rothemund Y, Preuschhof C, Bohner G, Bauknecht HC, Klingebiel R, Flor H, et al. Differential activation of the dorsal striatum by high-calorie visual food stimuli in obese individuals. Neuroimage 2007;37:410-21.

[70] Stoeckel LE, Weller RE, Cook EW, 3rd, Twieg DB, Knowlton RC, et al. Widespread reward-system activation in obese women in response to pictures of highcalorie foods. Neuroimage 2008;41:636-47.

[71] Porubska K, Veit R, Preissl H, Fritsche A, Birbaumer N. Subjective feeling of appetite modulates brain activity: an fMRI study. Neuroimage 2006;32:1273-80.

[72] Spiegler BJ, Mishkin M. Evidence for the sequential participation of inferior temporal cortex and amygdala in the acquisition of stimulus-reward associations. Behav Brain Res 1981;3:303-17.

[73] Kable JW, Glimcher PW. The neural correlates of subjective value during intertemporal choice. Nat Neurosci 2007;10:1625-33.

[74] Kosten TR, Scanley BE, Tucker KA, Oliveto A, Prince C, Sinha R, et al. Cue-induced brain activity changes and relapse in cocaine-dependent patients. Neuropsychopharmacology 2006;31:644-50.

[75] Bonson KR, Grant SJ, Contoreggi CS, Links JM, Metcalfe J, Weyl HL, et al Neural systems and cue-induced cocaine craving. Neuropsychopharmacology 2002;26:376-86.

[76] Tapert SF, Pulido C, Paulus MP, Schuckit MA, Burke C. Level of response to alcohol and brain response during visual working memory. J Stud Alcohol 2004;65:692-700.

[77] Naqvi NH, Rudrauf D, Damasio H, Bechara A. Damage to the insula disrupts addiction to cigarette smoking. Science 2007;315:531-4.

[78] Clifton PM, Keogh J. Metabolic effects of high-protein diets. Curr Atheroscler Rep 2007;9:472-8

[79] Pessoa L. To what extent are emotional visual stimuli processed without attention and awareness? Curr Opin Neurobiol 2005;15:188-96.

[80] Uher R, Murphy T, Brammer MJ, Dalgleish T, Phillips ML, Ng VW, et al. Media prefrontal cortex activity associated with symptom provocation in eating disorders. Am J Psychiatry 2004;161:1238-46.

[81] Amodio DM, Frith CD. Meeting of minds: the medial frontal cortex and social cognition. Nat Rev Neurosci 2006; 7:268-77.

[82] Gilbert SJ, Frith CD, Burgess PW. Involvement of rostral prefrontal cortex in selection between stimulus-oriented and stimulus-independent thought. Eur J Neurosci 2005;21:1423-31.

[83] Allan K, Rugg MD. An event-related potential study of explicit memory on tests of cued recall and recognition. Neuropsychologia 1997;35: 387-97.

[84] Lepsien J, Nobre AC. Attentional modulation of object representations in working memory. Cereb Cortex 2007;17:2072-83. 